\title{
Mechanisms of ischemic kidney tolerance in young and senescence-accelerated rats exposured to ischemic preconditioning or calorie restriction
}

\author{
Egor Plotnikov \\ A.N. Belozersky Institute of Physico- \\ Chemical Biology, Lomonosov Moscow \\ State University; \\ V.I. Kulakov National Medical \\ Research Center of Obstetrics, \\ Gynecology and Perinatology, \\ Moscow, Russia \\ plotnikov@belozersky.msu.ru \\ Irina Pevzner \\ A.N. Belozersky Institute Of Physico- \\ Chemical Biology, Lomonosov Moscow \\ State University, Moscow, Russia \\ irinapevzner@mail.ru \\ Denis Silachev \\ A.N. Belozersky Institute of Physico- \\ Chemical Biology, Lomonosov Moscow \\ State University; \\ V.I. Kulakov National Medical \\ Research Center of Obstetrics, \\ Gynecology and Perinatology, \\ Moscow, Russia \\ silachev_dn@belozersky.msu.ru
}

\author{
Nadezda Andrianova \\ Faculty of Bioengineering and \\ Bioinformatics, \\ Lomonosov Moscow State University, \\ A.N. Belozersky Institute of Physico- \\ Chemical Biology, Lomonosov Moscow \\ State University;Moscow, Russia \\ andrianova@belozersky.msu.ru \\ Ljubava Zorova \\ A.N. Belozersky Institute Of Physico- \\ Chemical Biology, Lomonosov Moscow \\ State University, Moscow, Russia \\ lju_2003@list.ru \\ Natalia Kolosova \\ Institute of Cytology and Genetics, \\ Siberian Branch of Russian Academy of \\ Sciences (SB RAS), Novosibirsk; \\ Russia, \\ kolosova@bionet.nsc.ru
}

\author{
Stanislovas Jankauskas \\ A.N. Belozersky Institute Of Physico- \\ Chemical Biology, Lomonosov Moscow \\ State University, Moscow, Russia \\ jankauskas.ss@gmail.com \\ Vasily Popkov \\ A.N. Belozersky Institute Of Physico- \\ Chemical Biology, Lomonosov Moscow \\ State University, Moscow, Russia \\ popkov.vas@gmail.com
}

Dmitry Zorov

A.N. Belozersky Institute of Physico-

Chemical Biology, Lomonosov Moscow State University;

V.I. Kulakov National Medical

Research Center of Obstetrics,

Gynecology and Perinatology,

Moscow, Russia

zorov@belozersky.msu.ru

\begin{abstract}
Calorie restriction (CR) and ischemic preconditioning (IPC) are the most efficient approaches ameliorating the severity of different pathological conditions. We investigated the protective potential of long-term calorie restriction and short ischemic preconditioning protocol in the model of acute kidney injury (AKI) in young Wistar and OXYS rats. In young rats, ischemic preconditioning, which consists of 4 cycles of ischemia and reperfusion alleviated kidney injury caused by 40-min ischemia. However, 6-month-old OXYS rats having signs of premature aging lost their ability to protect the ischemic kidney by IPC. However, CR of OXYS rats led to a significant decrease in creatinine and blood urea nitrogen levels after kidney ischemia, indicating significant protection.
\end{abstract}

Keywords - kidney, ischemia, preconditioning, aging, autophagy

\section{Introduction}

The phenomenon of improving of tissue ischemic tolerance by the preliminary impact of various stress factors is a universal intrinsic protective mechanism inherent to a large number of organs and tissues, including the kidney. Induced ischemic tolerance of the kidney is of great interest for nephrology, since activation of tissue resistance signaling pathways can be simulated using a number of pharmacological agents. Currently, a number of nephroprotective effectors have been identified, such as IPC and CR, which demonstrate a pronounced therapeutic potential in experimental studies [1,2]. However, their effectiveness has been studied mainly in young animals and not tested on old ones, which makes it difficult to translate these approaches to the clinic, since the main group of patients suffering from renal pathologies are the elderly.

This may be one of the reasons why most clinical trials have failed for drugs that have shown their effectiveness in in vivo experiments. However, working with old animals is associated with a number of objective difficulties, so an important task is to find models that allow us to study the phenomenon of aging in vivo. One of these models is a strain of OXYS rats that show signs of premature aging. A detailed study of OXYS rats revealed as early as during the first 12 months of life an early manifestation of diseases that are usually observed in old age: retinopathy, similar to agerelated maculodystrophy [3], osteoporosis [4], arterial hypertension and cardiomyopathy [5], sarcopenia [6], and neurodegenerative diseases, including Alzheimer's type [7]. It can be assumed that accelerated aging also affects the kidney, which makes these rats a unique model for studying the effect of aging on renal diseases.

The aim of this work was to study the effect of premature aging on the mechanisms of ischemic kidney tolerance.

\section{Materials and Methods}

The nephroprotective effect of CR and IPC in young and old animals in renal ischemia/reperfusion (I/R) was studied. The kidney was subjected to 40-min ischemia with preliminary IPC (4 cycles of 15 with ischemia and 15 with reperfusion) or CR. CR was performed for 4 weeks by limiting the amount of feed by $35 \%$ of the daily norm to $65 \%$ of the norm. To assess AKI severity, the concentration of serum 
creatinine and blood urea nitrogen (BUN) in the blood was measured, and the level of NGAL protein in the urine was determined.

\section{Results}

Kidney I/R led to the development of AKI in young Wistar rats and OXYS rats, 48 hours after ischemia, we observed an increase in the concentration of creatinine and urea in the blood, and the level of NGAL in the urine, while in OXYS rats the severity of renal failure was higher, as indicated by higher increase of serum creatinine and BUN after I/R. Both types of nephroprotective treatments reduced the severity of AKI in young animals, which was manifested in a decrease in serum creatinine and BUN. However, the effects on OXYS rats were rather different. In OXYS rats, ischemia after IPC keep causing a significant increase in AKI markers, meaning that the protective effect of this exposure was not occurred. Conversely, CR of OXYS rats led to a significant decrease in creatinine and BUN levels after kidney ischemia.

To analyze the mechanisms that may be responsible for the loss of efficiency of nephroprotection, the processes of autophagy and mitophagy in the kidneys of young and old animals in norm and after CR or IPC exposure were studied. Autophagy activation was observed in young animals after $\mathrm{I} / \mathrm{R}$ : the number of lysosomes increased in the proximal tubules (measured by LysoTracker Green staining), and the LC3I/LC3II ratio increased in the whole kidney homogenates. In rats, OXYS I/R did not lead to such changes. Measuring the LC3II/LC3I ratio and staining with a lysosomal probe showed that young animals after CR showed a more pronounced activation of autophagy compared to OXYS rats. The intensity of mitophagy, which was determined by the level of PINK-1 protein, was also higher in Wistar rats.

\section{Discussion}

A recent study has shown that the response of OXYS rats to fasting within 24 or 48 hours may differ in terms of expression of a number of genes associated with autophagy (Kozhevnikova et al, 2019). Furthermore, it was found that many effects coincide in direction, although gene expression differs in absolute values of increase/decrease. For example, after 24 hours of fasting, an increase in the expression of the Atg5, Becn1, and p62 genes was observed in both Wistar and OXYS rats, although the magnitude of the effect was different. We can assume that with prolonged dietary restriction, the effects of autophagy activation in OXYS rats may reach the threshold values necessary for adequate kidney protection. That is, despite the initial reduced effectiveness of autophagy and quality control pathways, long-term exposure compensates for this disadvantage. There is some evidence of this in the literature, since it was shown that in older animals with reduced autophagy efficiency, longer periods of CR had a more pronounced protective effect than short ones.

\section{Conclusion}

Thus, both protective approaches, CR and IPC, cause induction of ischemic kidney tolerance in young Wistar rats, but not in OXYS rats. Loss of efficiency of nephroprotection may be associated with disturbances of autophagy and mitophagy mechanisms. This ineffectiveness of autophagy may be critical for a short protective pulse (IPC), but prolonged activation stimuli (CR for a month) could induce it anyway.

\section{ACKNOWLEDGMENT}

The work was supported by the Russian science foundation grant \#18-15-00058.

\section{REFERENCES}

[1] Jankauskas, S.S.; Pevzner, I.B.; Andrianova, N.V.; Zorova, L.D.; Popkov, V.A.; Silachev, D.N.; Kolosova, N.G.; Plotnikov, E.Y.; Zorov, D.B. The age-associated loss of ischemic preconditioning in the kidney is accompanied by mitochondrial dysfunction, increased protein acetylation and decreased autophagy. Sci. Rep. 2017, 7, 44430

[2] Andrianova, N.V.; Jankauskas, S.S.; Zorova, L.D.; Pevzner, I.B.; Popkov, V.A.; Silachev, D.N.; Plotnikov, E.Y.; Zorov, D.B. Mechanisms of Age-Dependent Loss of Dietary Restriction Protective Effects in Acute Kidney Injury. Cells 2018, 7, 178.

[3] Markovets, A. M. et al. Alterations of retinal pigment epithelium cause AMD-like retinopathy in senescent-accelerated OXYS rats. Aging (Albany. NY). 3, 44-54 (2011).

[4] Muraleva, N. A., Ofitserov, E. N., Tikhonov, V. P. \& Kolosova, N. G. Efficacy of glucosamine alendronate alone \& in combination with dihydroquercetin for treatment of osteoporosis in animal model. Indian J. Med. Res. 135, 221-227 (2012).

[5] Bobko, A. A. et al. 19F NMR measurements of NO production in hypertensive ISIAH and OXYS rats. Biochem. Biophys. Res Commun. 330, 367-370 (2005).

[6] Vays, V. B. et al. Antioxidant SkQ1 delays sarcopenia-associated damage of mitochondrial ultrastructure. Aging (Albany. NY). 6, 140148 (2014).

[7] Stefanova, N. A., Muraleva, N. A., Skulachev, V. P. \& Kolosova, N. G. Alzheimer's disease-like pathology in senescence-accelerated OXYS rats can be partially retarded with mitochondria-targeted antioxidant SkQ1. J. Alzheimer's Dis. 38, 681-694 (2014). 